\title{
USABILITY ENGINEERING \\ IN THE PROTOTYPING PROCESS \\ OF SOFTWARE USER INTERFACES \\ FOR MOBILE MEDICAL \\ ULTRASOUND DEVICES
}

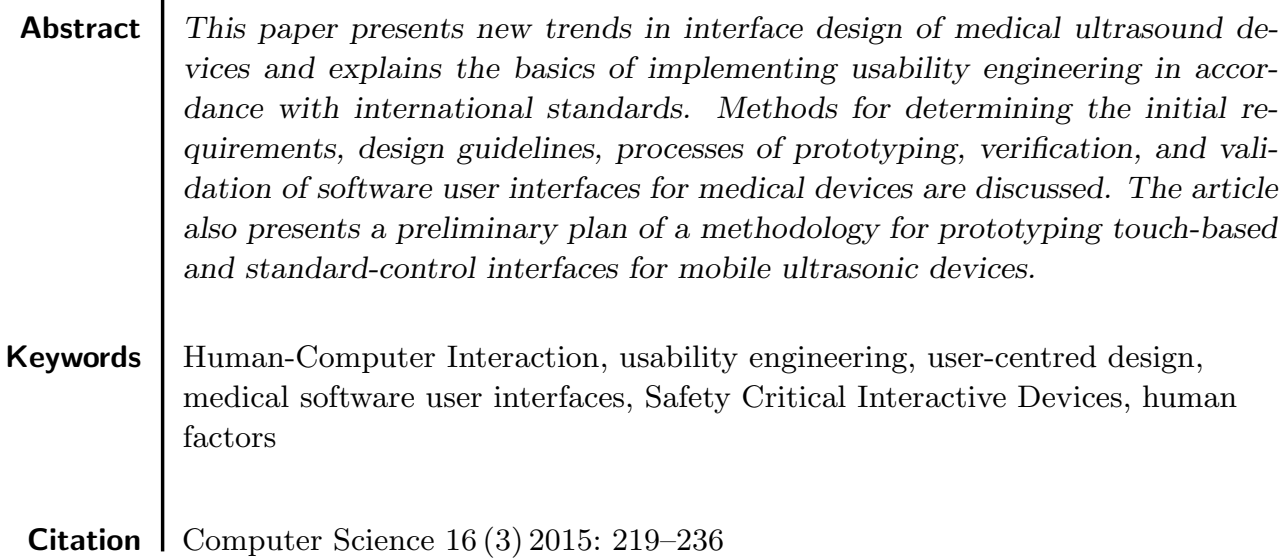




\section{Introduction}

The extremely fast development of information technology has had a definite impact on the evolution and expansion of new interactive systems that are used in an increasing number of areas of modern life. The technical sophistication and number of features that such systems offer entail the need for proper analysis, research, and design of interaction between the user and the computer system. These tasks are the foundation of the Human-Computer Interaction discipline. Well-designed interactive solutions that guarantee a high level of usability as well as ergonomic and functional quality have become increasingly important factors to consider when choosing an interactive system. These qualities allow users to efficiently, effectively, comfortably, and (very important for medical devices) safely operate with medical products. Systems built according to HCI methodologies should allow us to achieve a quality User-Experience and shape positive public opinion, which usually has a great impact on the market success of a product.

Naturally, low usability of products is a very common cause of their market failure. Users are dissatisfied with the usage and generally rate it bad. In the end, it gives poor sales and low profit to a developer. In the worst case, it causes a total loss, resulting in the need to withdraw the product from the market. It also generates additional costs in dealing with dissatisfied users, complaints, and the necessity to redesign an existing product/service/system. This situation significantly weakens the image of and confidence in the brand.

For medical device manufacturers, the stakes are much higher. For example, based on the methods how medical device interface informs the user about a critical event and what action is proposed, human health and life could be endangered. Numerous studies have confirmed that low usability of medical device interfaces has a significant impact on the growth of the use errors, and it is a threat to patients [3]. This has led organizations such as the Food and Drug Administration (FDA), International Electrotechnical Commission (EIC), and the International Organization for Standardization (ISO) to make the following guidelines and standards concerning the process of implementing usability engineering in the design of medical device interfaces.

\section{Review of the latest trends in interface design for medical ultrasound devices}

The percentage of medical devices where interaction takes place through software user interfaces is also increasing. This situation is mainly caused by lowering the cost of production of devices based on microprocessors and growing market demand. There are new opportunities in this area; for example, enriching the functions of the device by updating the operating system without the need of expensive replacement of components. This is of great economic importance to manufacturers. The popularity of modern mobile devices such as smartphones or tablets has also had a big 
impact, which resulted in the emergence of new methods of interaction and possibilities. Many users have become accustomed to the flexible adjustment of appearance and features of the user interface to suit their needs. Regardless of the complexity of the medical device interface, it should facilitate user tasks, protect against errors, and satisfy customers. The examples presented below are proof that the design of medical ultrasonic equipment changed dramatically and became similar to modern consumer multimedia devices.

\subsection{MobiSante MobiUS SP1 and MobiSante MobiUS TC2}

MobiUS SP1 (see Fig. 1) uses a Toshiba TG01 smartphone running under Windows Mobile 6.5. The device does not have the typical features of a mobile phone, but thanks to the Wi-Fi module and GSM communication, it can (for example) share and transfer saved photos to the base hospital for further consultation/archiving. The device also saves images to the memory card. It can be also connected to a computer. The small size makes it ultra-mobile, and its relatively low cost can certainly affect the popularization of this type of equipment. Interface solutions recall mobile operating systems - large buttons and icons on the touchscreen are easy to read and use.

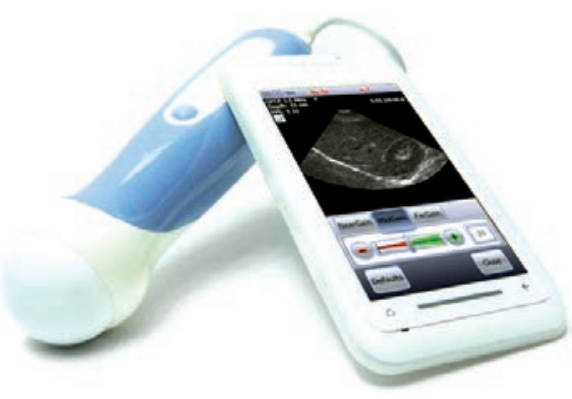

Figure 1. MobiSante MobiUS SP1 ${ }^{1}$.

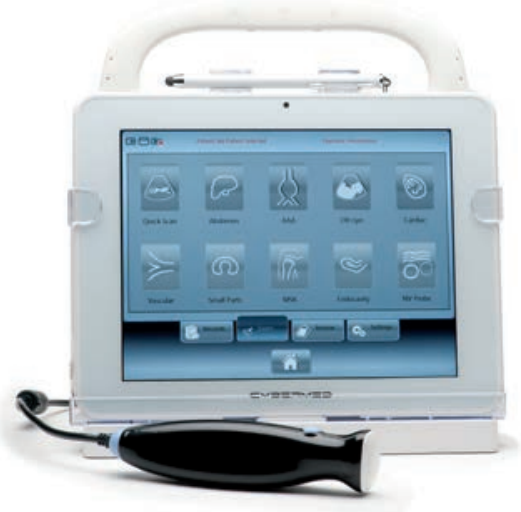

Figure 2. MobiSante MobiUS TC2 ${ }^{2}$.

MobiUS TC2 (see Fig. 2) uses a tablet instead of a smartphone, which offers a larger workspace.

\subsection{GE Healthcare ${ }^{T M}$ S Vscan}

As opposed to the MobiSante MobiUS SP1, this device does not rely on a smartphone, and has been designed from the ground up by GE (see Figs 3, 4).

\footnotetext{
${ }^{1}$ source: http://www.mobisante.com/products/product-overview/

${ }^{2}$ source: http://www.mobisante.com/products/tc2/
} 
With intuitive user interface solutions, the device can be operated with only the thumb of one hand. The control panel consists of a small number of buttons and is touch-sensitive, which could be helpful for regulations of specific parameters.

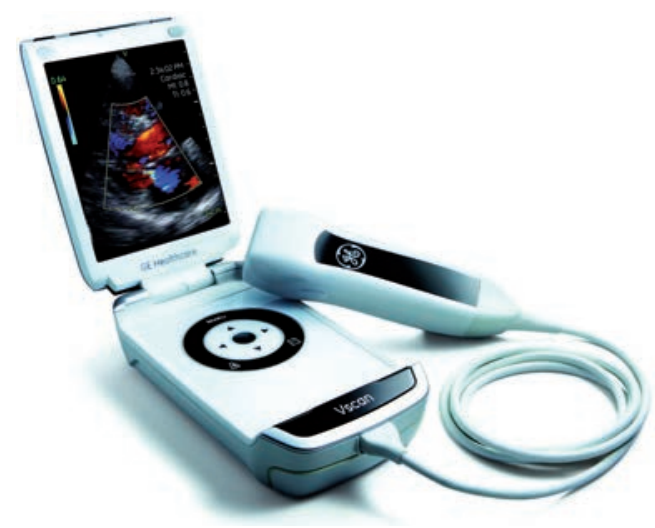

Figure 3. GE Healthcare ${ }^{\mathrm{TM}} \mathrm{s} \mathrm{Vscan}^{3}$.

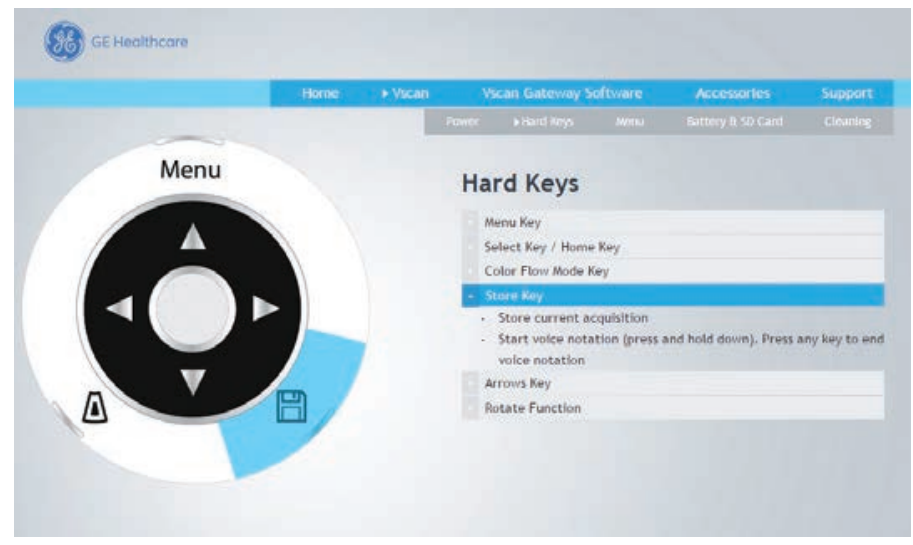

Figure 4. GE Healthcare ${ }^{\mathrm{TM}} \mathrm{s}$ Vscan tutorials ${ }^{4}$.

In addition, the manufacturer offers very extensive educational materials on the website, consisting of visual guides, interactive instruction, and simulations. These help to quickly familiarize users with its operations.

\footnotetext{
${ }^{3}$ source: https://vscan.gehealthcare.com/about-uses-us-en

${ }^{4}$ source: https://vscan.gehealthcare.com/vscan/vscan/hard-keys
} 


\subsection{Sonosite S Series Ultrasound System}

SonoSite S Series devices (see Fig. 5) can be mounted on a wall, ceiling, or wheelchair, making them portable and convenient to use in various places. The device is controlled by knobs and buttons located near the monitor (the operation of which depends on the context of use) and is configurable. This allows users to adjust the operation of the interface elements to their individual needs, providing abundant flexibility when operating the device.

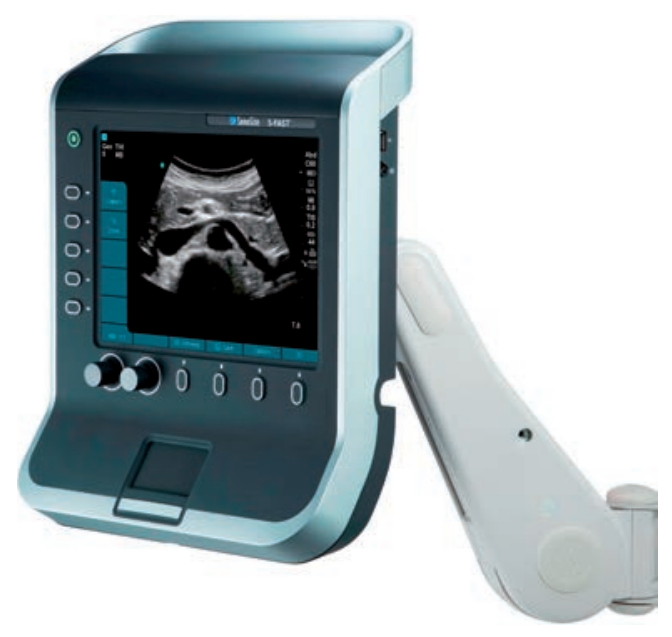

Figure 5. Sonosite S Series Ultrasound System ${ }^{5}$.

\subsection{Siemens ACUSON Freestyle Ultrasound System}

This device (see Fig. 6) is the first ultrasound that uses wireless connectivity with the probes (up to 3 meters away) by using a range of $8 \mathrm{GHz}$ Ultra-Wideband (UWB). UWB is a technology for the transmission of data using techniques which cause the spreading of radio energy over a very wide frequency band, with a very low power spectral density. It limits the interference potential with conventional radio systems, and the high bandwidth can allow very high data throughput for communications devices as well as high precision for location and imaging devices.

The device and probes use a variety of built-in antennas to achieve the best quality of the transmitted image. Bluetooth technology is used for bi-directional communication between the console and the probes as well as the location and positioning of the probe. The main control panel is located under the monitor and provides soft key buttons, trackball buttons, knobs, and an optional USB mouse. Probes and battery supplies can be sterilized to prevent infections.

\footnotetext{
${ }^{5}$ source: http://www.sonosite.com/au/ultrasound-products/s-series
} 


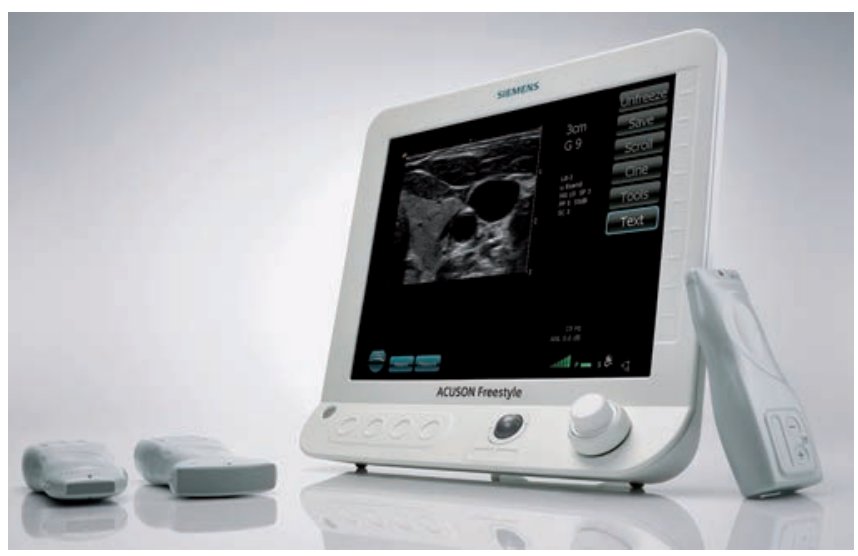

Figure 6. Siemens ACUSON Freestyle Ultrasound System ${ }^{6}$.

\subsection{SonoSite X-Porte}

The device (see Fig. 7) acts as an "all-in-one kiosk", with a minimalist design that ensures a high level of ergonomics and ease of everyday use. It features a new beamforming technology - Extreme Definition Imaging (XDI), a reduction of noise and artifact signals from the probe, and an innovative and intuitive user interface. The unit consists of two screens - the top displays images from the probe, and the bottom serves as a touch panel.

The bottom touch panel allows users to adjust settings like type of study, probes, and imaging. It also makes it possible to enter patient data, start an examination, access educational materials, etc. To navigate through the interface, popular multi-touch controls and gestures similar to those from smartphone/tablet devices are utilized. The unit has no physical buttons or controls - only a screen keyboard is available. These solutions are easy to keep clean and have a low risk of transferring infections. In addition to the stationary version of the device, the manufacturer has also provided the option to disconnect the main unit to facilitate operation as a portable device.

The designers have focused much attention to aspects of User Experience to ensure the most friendly and easy-to-use user interface (see Fig. 8) for comfortable and optimal operation in different environments. The device is dedicated to both beginners and advanced users. The touch panel screen layout can be adapted to the needs and requirements of the user. This allows for to changing the order of items on the menu and displaying only those options that are most commonly used, for example.

\footnotetext{
${ }^{6}$ source: http://www.healthcare.siemens.pl/ultrasound/ultrasound-point-of-care/ acuson-freestyle-ultrasound-system
} 


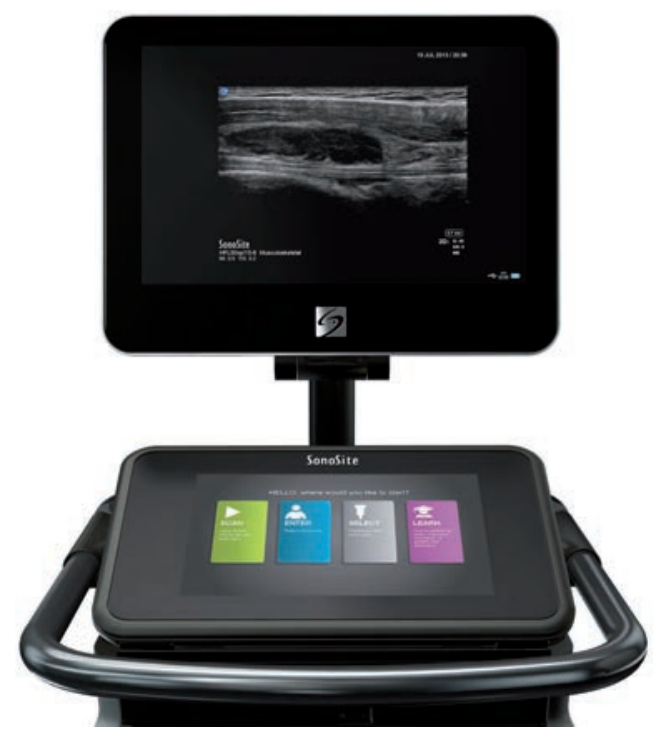

Figure 7. SonoSite X-Porte ${ }^{7}$.

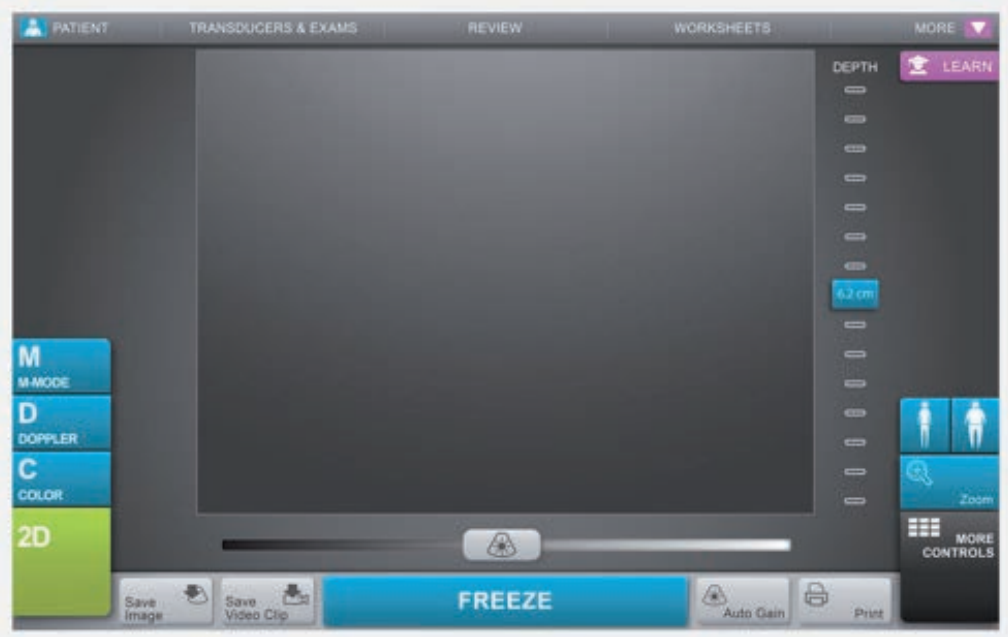

Figure 8. SonoSite X-Porte user interface ${ }^{7}$.

The small width of the unit makes it easy to move even in tight spaces. Thanks to the possibility of closing the main screen, the device is more compact and is protected from accidental damage during transport.

\footnotetext{
${ }^{7}$ source: http://www. sonosite.com/products/x-porte
} 
With the help of experts from the medical education department, the manufacturer has developed educational materials that are an integral part of the system. Users can find guides and tutorials concerning medical examination and use of the device. Noteworthy is the fact that they are available even while using the probe and offer real-time 3D animations presenting step-by-step instruction throughout the whole procedure. This can be helpful for less-experienced users and provide greater productivity (implementing "just-in-time learning"). It also accelerates the process of teaching novice users how to work with the device.

\subsection{Philips Sparq}

This is another ultrasound device (see Fig. 9) whose compact design allows us to classify it within the "point-of-care" (POC) segment, which means that the medical equipment can be delivered to the patient's bed for the duration of examination and is easily transported to new places. The large 17-inch screen mounted on a movable arm has a positive effect on the clarity and visualization of the presented data and can be useful in various conditions of usage. The device uses SonoCT technology, which reduces image artifacts from the probe, and XRES technology, which removes interference and ensures greater readability of the signal. The manufacturer decided to use a flat-glass surface with no physical buttons, which reduces the likelihood of infection and provides easier cleaning of the equipment.

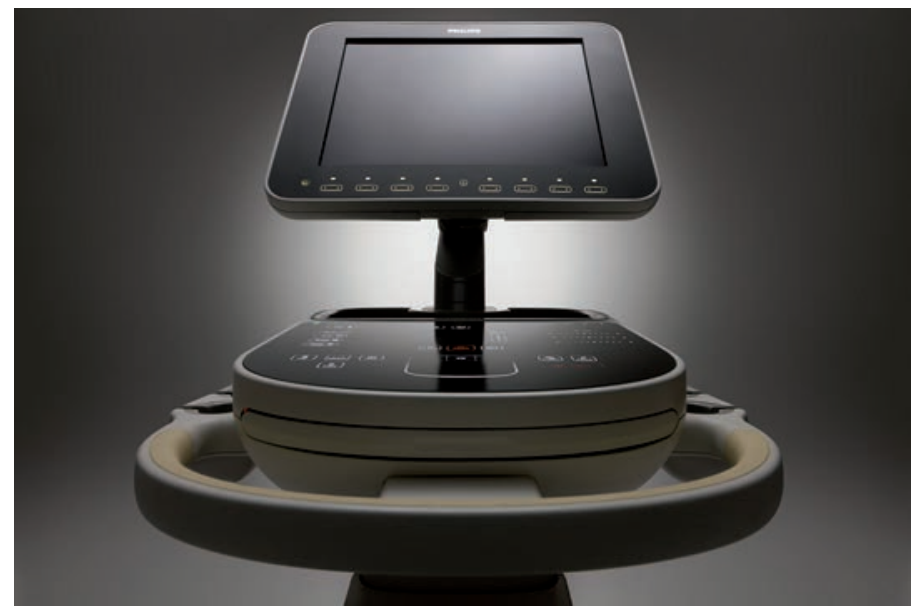

Figure 9. Philips Sparq

The dynamic and intuitive interface sans physical buttons is easy to learn and use (see Fig. 10). For novice users, the interface offers an Autoscan function that operates by pressing a single button. It allows the user to automatically identify the type of tissue and adjust the picture quality from the probe without manual control. This radically reduces time spent on adjusting settings before and during the exami- 
nation. The device provides a set of guidelines for the examination (SmartExam) and automates system functions to suit current needs. Simplicity Mode allows the user to leave active (visible) only those options in the control panel that are most-commonly used.
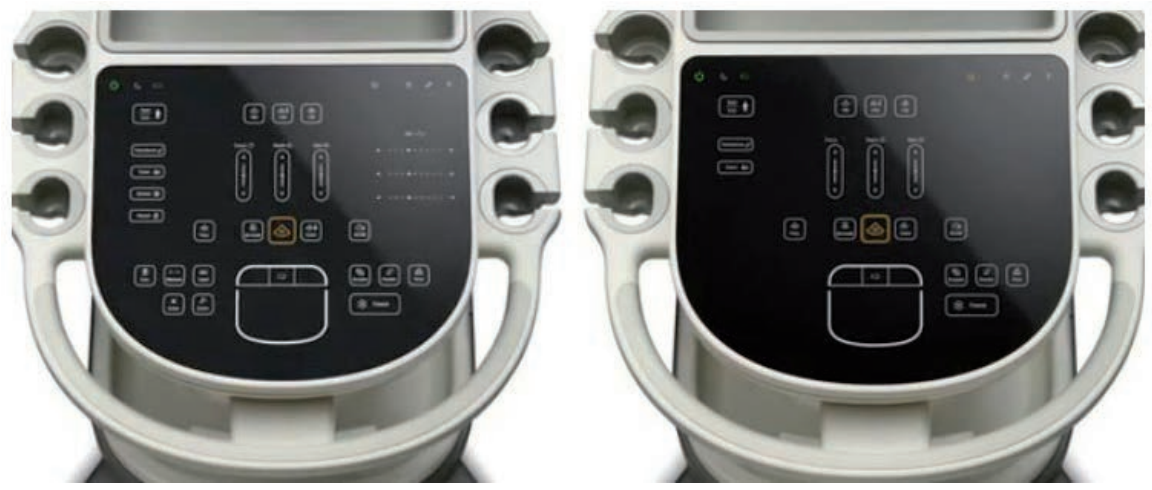

Figure 10. Philips Sparq control panel ${ }^{8}$.
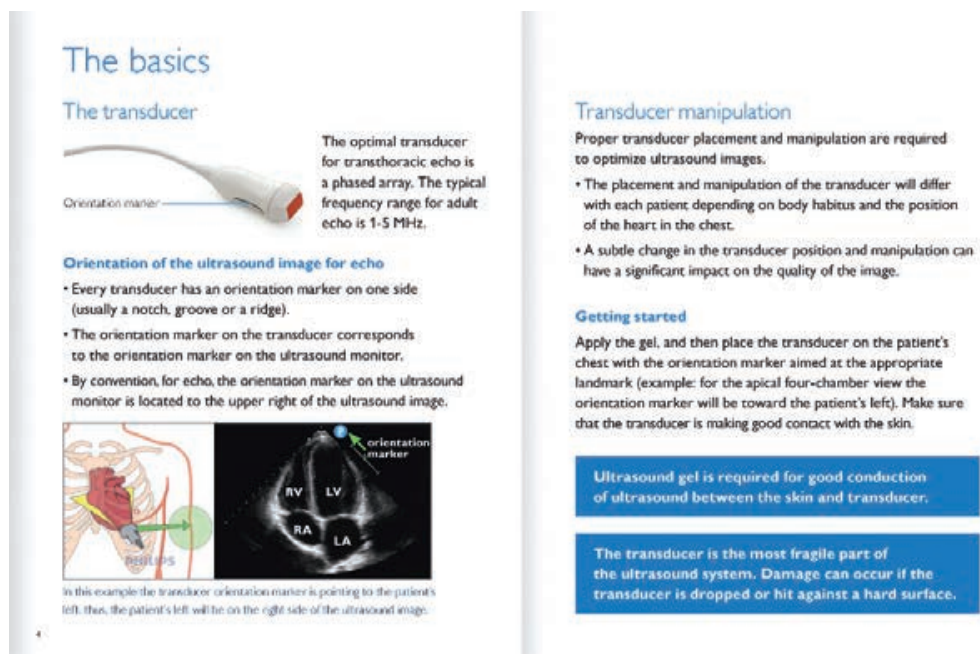

Figure 11. Philips Ultrasound Education (screenshot of iPad application).

The system guides the user through the steps of the examination process, which is a great help for less-experienced and new customers. Starting the examination is also possible by scanning the corresponding barcode that is assigned to the patient in the hospital. After the test, a one-page report with an analysis of results is prepared,

\footnotetext{
${ }^{8}$ source: http://www.healthcare.philips.com/main/products/ultrasound/systems/sparq/
} 
which can be transmitted wirelessly via a DICOM network to provide information and archive documents.

The device also offers specific support during biopsies in the form of needleposition visualization, which is very important to accurately perform the examination and contributes to the safety of the patient.

The manufacturer provides detailed instructions on working with ultrasound devices, through video tutorials and guides available on the website and in the form of interactive textbooks available for mobile platforms as free applications (see Fig. 11). This means they can be easily updated and are always at hand. Legible and userfriendly forms of presentation are richly illustrated, making it more conducive to easy learning.

\section{Basics of the standard of usability engineering for medical devices}

According to the ISO 9241 standard, usability is defined as the product ${ }^{\mathrm{TM}_{\mathrm{S}}}$ attribute specifying the ease of use. It is described by the measure of effectiveness (can the goal of the user be fully achieved?), efficiency (what is the cost of achieving the goal?), and satisfaction (which emotions and/or reactions are triggered in user interaction with the device?). Usability engineering is a sub-area of ergonomics - an interdisciplinary science concerned with the adaptation of working conditions to human capabilities. It is associated with other sciences, including psychology, human factors, anthropology, etc.

The standard ISO/IEC 62 366: Medical Devices - Application of Usability Engineering to Medical Devices replaced the older ISO/IEC 60601-1-6: Medical electrical equipment - Part 1-6: General requirements for safety - Collateral standard: Usability. The new standard requires that the manufacturers of medical devices deploy Usability Engineering Process based on a User-Centered Design. This standard applies not only to the device itself, but also to documentation and training materials associated with the device. The new norm extends to all medical devices (previously it was applied only to electrical medical devices). The standard can also be a guide in the process of implementation of the principles of usability, because it contains many annexes with detailed examples of the application of the methods.

Ensuring compliance with the standard in order to implement usability engineering is a process where technical skills are only a part of a bigger image. It is an interdisciplinary approach consisting of various stages: design, evaluation, testing, and validation of the proposed solutions. The manufacturer must carefully determine how to ensure high usability and how to minimize the risks associated with interaction with the system.

According to the above standard, the implementation of usability engineering process for medical devices is composed of several stages: 
- Application of the medical device; it defines the key features associated with the use of devices ,such as intended medical indication, population and profile of the patients, context of use, and basic operating principle.

- Identification of frequently-used functions of the device; it allows us to pay special attention to these features that, if designed properly, could significantly reduce operating errors.

- Identification of risks and hazardous situations associated with usability; it assumes the analysis of characteristics of the device in terms of impact on the risk of misuse and allows the identification of existing and anticipated risks associated with interaction with the device's interface.

- Identification of the primary operating functions of the device; it concerns features which have a direct impact on the safety of the device, including the previously-mentioned frequently-used functions.

- Developing usability specification. This step defines the testable requirements for verification of the usability of the interface. For this purpose, use cases and goals that must be achieved by the end user should be prepared. It also allows us to check for possible difficulties in understanding the basic functions of the device.

- Preparation of usability validation plan; this defines the methods and criteria for evaluation and validation of the primary functions of the device in terms of the predetermined usability specification. It is also necessary to determine a representative group of users of the device. Validation methods can be both qualitative and quantitative. Tests should be carried out in a laboratory environment that simulates real environments.

- Design and implementation of the user interface; this is the iterative process of user interface design, including frequent verification that takes place during the whole development cycle.

- Verification of the user interface usability. This process is carried out on the basis of a predetermined specification of usability. It can be based on user tests and heuristic evaluation.

- Validation of the medical device usability. This task takes place at the last stage of development and assumes that the usability test will be conducted based on a validation plan. The validation should be carried out by people who are not directly involved in the design and development of the user interface. In the case of a negative assessment, the manufacturer must redesign the device and repeat the processes of verification and validation.

The steps presented above concerning implementation of the usability engineering process must be documented in the Usability Engineering File. The structure of this document is presented in ISO/IEC 62366 standard [4]. It is proof that the manufacturer complies with the guidelines described in the standard, and it provides an effective audit of the entire process. The process itself and its elements are iterative. 


\section{Methods for determining the initial requirements for a medical device user interface}

Teams that are responsible for the design and construction of a new medical device must work in parallel on the development of the technology and the implementation of the interface. Requirements related to usability should be a part of the functional and technical specification of the device. Unsynchronized cooperation between experts of different fields often leads to the necessity of making changes, which are especially costly if introduced in the late development stages of the project. According to the above-mentioned standard, it is necessary to determine requirements related to the primary functions and applications of the device, the context of its use, the requirements of users, patient population, and potential risks arising from improper interaction with the interface. There are many helpful methods, such as:

- using storytelling for initial requirements elicitation,

- focus groups (consisting of future device users, experts, and developers),

- participatory observation of users in their real working environment,

- cognitive walk-through (usability experts run through specially-designed tasks within the interface).

The proposed methods allow for the better understanding users, who often have problems with articulation of which features they really need and how they expect to interact with the interface.

The designers of software user interfaces for medical devices must also be aware of the initial conditions that are independent of user requirements. One of the main criteria that influence the design of software user interfaces is the functional complexity of medical devices. The size of the device is also important, mostly because it affects the size of the interface screen. Large displays provide more information on one screen, while the smaller ones force designers to use a series of related screens for this purpose. Another hardware factor is the type of power supply; the device can be permanently connected to AC power or be battery operated. This may determine the type of display technology.

Development tools used for implementation may have an impact on the design of medical interfaces. This explains the similarity of some interface elements to those known from popular operating systems. The positive side of this process is production cost savings and the ability to utilize user habits. The negative side is further propagation of the shortcomings of existing interfaces as well as the lack of optimization of specific requirements for medical devices.

\section{Guidelines for the design of software user interfaces for medical devices}

Guidelines concerning the design of software user interfaces for medical devices are largely based on norms and standards concerning Human-Computer Interaction. The 
literature [8] presents very precise specifications and requirements for the various elements of the user interface. The most important aspects essential to the process of determining guidelines for the medical device are as follows:

- Conceptual model. This should be created at the beginning of the development process of the interface; it allows determining how the target audience imagines interacting with the device. Such a model should assume the use of a minimum number of features and components necessary to operate the device. The interface model should focus on the user task rather than the internal structure and logic of the device.

- Structure of the interface; it should depict the conceptual model and be in line with the perceptual abilities of users. Particularly noteworthy is the screen hierarchy and navigation through it, which should support the user in finding critical and frequently-used functions.

- Style of interaction; this has a very large impact on the intuitiveness and efficiency of the user interface. In many cases, the type of interaction depends on the chosen technological solutions. For these reasons, the decision of choosing the style of interaction should be taken at an early stage of development of the interface concept. The interaction style should be tailored to the context of its use and the aspects of ergonomics.

- Layout of screen; it determines not only aesthetic values but also the quality of interaction with the device. It is difficult to describe one specific pattern that has proven to be good in every situation. Therefore, it is important to take into account basic guidelines related to the design of a useful user interface, then make several prototypes and test them in order to find the best solution.

- Legibility of interface elements; misreading critical information displayed on the screen may have very serious consequences for the patient. Because of this, it is necessary to ensure high readability of both text elements and graphics.

- Aesthetics of the interface; this has a positive influence on the assessment of the device, and it helps users focus on the task being performed. Therefore, the process of designing a graphical user interface should incorporate people having experience in the field of industrial design.

- Data entry. This task should not only be easy and efficient, but also must guarantee that the data will be made accurate, complete, and presented in the correct order.

- Color of the interface; this is important for both the functional and aesthetic quality of the interface. The proper use of color draws attention to important elements and determines the readability of visual aspects of the user interface. The key is to follow accepted conventions of encoding information using color for medical devices. Color should not be used as the only factor responsible for information coding due to those users with impaired color vision.

- Display of dynamic data. Some values must be monitored and displayed in real time; it is necessary to determine the details of their animation, refresh rate, signal changes, and showing emergencies. 
- Interactive mechanisms. The interface can be controlled by different mechanisms: buttons near the screen, knobs, touch screens, keyboards, keypads, etc. The method of control should be ergonomically designed and adapted to the conditions of use of the device.

- Speech-emitting user interfaces. User testing of speech prompting is advisable with special attention to tone of voice, delivery rate, volume, conciseness, and repetition.

- User support. Due to the fact that users may differ in terms of knowledge about how the device works, support materials and easily-accessible assistance should be provided. It may take the form of a dialog system, information strips, or additional help sections where the user can find simplified instructions. Worth considering is the use of graphic descriptions or animations, which in some cases are easier in perception.

\section{Process of prototyping software user interfaces for medical devices}

The predefined requirements and guidelines should be used during the process of prototyping interfaces. In the early stages of prototyping, standard methods such as paper prototypes (that allow determining layout of the interface and the basic concepts of interactions) and sorting cards (that help the designer to define the logic and structure of the menu) may be good solutions. The next step is to build an interactive mock-up that allows for pre-verification and assessment of the level of ergonomics/usability of interaction with the device as well as the identification of potential sources of error. For this purpose, the designed interfaces are subjected to initial usability testing. If there is a need to carry out tests with doctors and medical staff, the proposed prototypes should be really similar to the target interfaces in terms of visual and interaction aspects. It is crucial to optimize the process of user interface prototyping because, at this stage, a number of key changes may be introduced that, in turn, lead to the need of frequent retests. An important element of this optimization is to choose efficient and effective technology for building prototypes. This has a huge impact on the speed and ease of construction of such interactive mock-ups. It is also responsible for particular fidelity level of appearance and interaction with the prototype.

\section{Processes of verification and validation of software user interfaces for medical devices}

Usability standards require regular verification and validation of medical devices. Verification applies to individual elements and features of the device at different stages of product development. In order to verify a software user interface, many common methods can be used: heuristic evaluation, inspection of key features, cognitive walkthrough, simple tests with users, etc. [5]. 
Validation, however, is the final stage of development and allows assessment of the medical device as a whole in terms of its usability and safety. For this purpose, usability tests are carried out in a specially-prepared laboratory, which should offer conditions similar to the real environment where the device will be used. The laboratory is usually equipped with cameras, microphones, and usability-testing software that allow for the recording of user behavior and interaction with the user interface by using screen capture. It is recommended that the test moderators be present in an adjacent room, separated by a semi-transparent mirror. Installed recording equipment should not distract participants. Popular professional programs for image acquisition, registration events, and analysis of test data are (for example) Noldus ObserverXT, TechSmith Morae, etc.

In the case of testing the interfaces based on mobile devices, additional applications may be helpful for cloning device screens in real time on a computer using Wi-Fi or web services that collects and analyzes events occurred in the user interface.

The test should be conducted with the involvement of representatives of the user group and use a representative task. In the usability testing of standard interfaces, it is assumed that only six users are sufficient to detect $80 \%$ of the potential usability problems. In the case of medical device interfaces, it is recommended to test the safetycritical functions on a group of ten users who were carefully selected [5]. Although laboratory conditions will never be identical to real testing, this form of testing has many advantages. It allows repeated performance of even unlikely scenarios with different users in controlled conditions with no risk to patients. Results collected during testing should be carefully examined by experts and available to the project group. In accordance with the usability standard, the test results must be documented in the Usability Engineering File.

\section{User-centered design methods may be insufficient for complex mobile medical interfaces}

In 2007, the European Council revised the Medical Device Directive to include the provision that software can be classified as a medical device. This amendment came into effect in March, 2010. In result, software can be subjected to the same standards as regular medical devices [2].

In 2010, the Food and Drug Administration (FDA) pointed out 23 medical devices that were classified as Class I, (reasonable probability that use of these products will cause serious adverse health consequences or death). It was found that of these recalls, 6 were caused by software problems [6].

These problems show that typical approaches of HCI (like User-Centered Design) are very important to create useful interfaces, but may be insufficiently powerful to solve all of the problems of interaction in the safety-critical context of medical devices [7].

Additionally, existing usability standards do not satisfy the measuring of qualityin-use of mobile user interfaces in the healthcare area [1]. 


\section{Contribution to the problem solution and collaboration}

Contribution to presented problems lies in the proposal of a new methodology for prototyping software user interfaces for mobile ultrasonic devices. The proposed methodology is based on the selected HCI concepts and usability standards for medical devices and will be verified in practice by its application to specific cases of various types of interfaces for mobile ultrasound devices. Touch-based and standard-control interfaces will be taken into account. Additionally, tests of alternative methods of controllers (like a haptic device) are planned. The main objective is to determine which of the proposed ideas have a particular impact on the level of usability and safety of mobile ultrasound medical devices focusing on different types of interfaces and interaction methods.

The devices will be constructed in Institute of Fundamental Technological Research, Polish Academy of Sciences in Warsaw.

Preliminary plan of new methodology consists of:

- Requirements Phase:

- The aim of this process is to define domain-based context of the usability requirements relating to mobile ultrasonic device with touch screen vs. standard control.

- It will be based on usability heuristics and analyzing existing devices.

- User Modeling Phase:

- In this phase, the stereotypes of the users will be recorded.

- It will be based on an empirical study with doctors using previouslyconstructed devices by observing usage in situ.

- Storytelling will be used that can serve as a robust self-report method by supporting the retrieval of contextual cues from long-term memory.

- Analysis Phase:

- Based on the characterization of ultrasonic device context from Phase 1 and user stereotypes developed in Phase 2, a set of context descriptors will be proposed.

- Context Descriptor is a user requirement that will form the basis for mobile user interface adaptation in the context of touch screen vs. standard control.

- Design and Experimental Phase:

- Based on Context Descriptors, new features/changes of user interfaces will be adopted to extend existing requirements.

- Prototypes of user interfaces (touch and standard-control versions) will be developed and connected to an existing device.

- Experiments with other types are planned (i.e., haptic device)

- Testing and Validation Phase:

- Developed versions of interfaces will be tested with doctors in a controlled environment (usability lab).

- Subjective Factor Analysis in context of touch and standard-control versions: 
- learning the device,

$\circ$ using the device,

o performing a particular task,

$\circ$ finding features,

$\circ$ understanding navigation,

o recovering from error.

- Quality-in-use factors which will be measured:

$\circ$ effectiveness,

o productivity,

$\circ$ task efficiency,

o safety (error prevention and error recovery),

o task navigation (cognitive load).

\section{Conclusions}

The introduction of standardization in the design process of software user interfaces for medical devices primarily improves the usability and safety of this equipment, and can also bring many benefits to the manufacturers of medical devices. Project groups responsible for the creation of interfaces must follow the required steps of implementation of usability engineering and find ways to optimize the different stages of the process, especially concerning prototyping and user-driven development.

However, the proposed methodology may be helpful for designers and doctors in improving the designing process of mobile ultrasonic devices. Differences between touch versions vs. standard-control versions and alternative methods of controls may produce interesting observations based on context of use. Future articles will discuss results of this research in detail.

\section{Acknowledgements}

I would like to thank Prof. Krzysztof Marasek and Dr. Marcin Lewandowski for their support. This work was funded by a grant agreement no. ST/MN/MUL/2013 at the Polish-Japanese Academy of Information Technology.

\section{References}

[1] Alnanih R., Ormandjieva O., Radhakrishnan T.: A New Methodology (CONINFO) for Context-Based Development of a Mobile User Interface in Healthcare Applications. In: A. Holzinger, M. Ziefle, C. Röcker, eds., Pervasive Health, pp. 317-342, Springer, London, 2014.

[2] Council Directive 2007/47/EC (Amendment). Official Journal of The European Union. European Council, Luxembourg, 2007.

[3] Hodgson P.: Usability for Medical Devices: A New International Standard, http: //www.userfocus.co.uk/articles/IS062366.html. 
[4] ISO/IEC 62 366: Medical Devices - Application of Usability Engineering to Medical Devices.

[5] Matern U., Büchel D.: Usability of Medical Devices. In: R. Kramme, K. P. Hoffmann, R. Pozos, eds., Springer Handbook of Medical Technology, pp. 59-71, Springer, Berlin, Heidelberg, 2011.

[6] Sandler K., Ohrstrom L., Moy L., McVay R.: Killed by Code: Software Transparency in Implantable Medical Devices. Software Freedom Law Center, New York, USA, 2010.

[7] Thimbleby H.: User-Centered Methods Are Insufficient for Safety Critical Systems. In: H. Andreas, ed., HCI and Usability for Medicine and Health Care, Lecture Notes in Computer Science, vol. 4799, pp. 1-20, Springer, Berlin, Heidelberg, 2007.

[8] Weinger M. B., Wiklund M.E., Gardner-Bonneau D. J.: Handbook of Human Factors in Medical Device Design. CRC Press, Boca Raton, FL, USA, 2011.

\section{Affiliations}

\section{Marcin Wichrowski}

Polish-Japanese Academy of Information Technology, Warsaw, Poland, mati@pjwstk.edu.pl

Received: 19.01 .2015

Revised: 23.03.2015

Accepted: 23.03.2015 